\title{
Enhanced Implementation of the Complex Images Method to Study Bound and Leaky Regimes in Layered Planar Printed Lines
}

\author{
Raúl Rodríguez-Berral, Francisco Mesa, Member, IEEE, and Francisco Medina, Senior Member, IEEE
}

\begin{abstract}
The discrete complex image technique, originally developed to deal with three-dimensional planar structures, has been recently applied to two-dimensional planar and nonplanar transmission lines embedded in layered substrates. In spite of successive refinements, some important practical issues concerning the sampling path, treatment of spectral Green's functions poles, and extension to the leaky regime are still open and deserve deeper examination. It will be shown that a suitable choice of the sampling path is key to reducing the number of images and to avoid the necessity of extracting spectral poles when the bound regime is considered. The situation arising from the analysis of the leaky regime becomes more complex and the adequate choice of the sampling path, as well as an appropriate pole extraction, turn out to be essential. In particular, a new pole-extraction strategy able to deal with poles associated with both proper and improper modes of the background waveguide is proposed. The advantages of using the theory in this paper will be illustrated by diverse numerical results for the bound/leaky regime of various planar lines. Finally, the suitability of the method for covered planar lines will be demonstrated.
\end{abstract}

Index Terms-Complex images, layered substrates, pole extraction, printed lines.

\section{INTRODUCTION}

A S IS WELL known, the underlying idea of the discrete complex image technique (DCIT) is to approximate the spectral-domain Green's functions as an expansion of complex exponentials whose spatial counterpart is known in closed form. This method was originally developed to obtain the three-dimensional (3-D) spatial Green's function for the vector and scalar potentials of dielectric layered media [1]-[3]. Later, Kipp and Chan [4] introduced pertinent corrections to the formulation in [1] and, further, these same authors [5] and Aksun [6] proposed a robust approach based on a two-level approximation of the Green's functions in the spectral domain. As in the 3-D case, the advantages of the DCIT approach in the two-dimensional (2-D) frame rely on the possibility of avoiding the time-consuming Sommerfeld-type integrals required by the application of the method of moments for solving the

Manuscript received July 26, 2003; revised September 24, 2003. This work was supported by the Spanish Ministry of Science and Technology and FEDER funds under Project Comisión Interministerial de Ciencia y Tecnología TIC2001-3163.

R. Rodríguez-Berral and F. Mesa are with the Grupo de Microondas, Departamento de Física Aplicada 1, Escuela Técnica Superior de Ingeniería Informática, Universidad de Sevilla, 41012 Seville, Spain (e-mail: mesa@us.es).

F. Medina is with the Grupo de Microondas, Departamento de Electrónica y Electromagnetismo, Facultad de Física, Universidad de Sevilla, 41012 Seville, Spain (e-mail: medina@us.es).

Digital Object Identifier 10.1109/TMTT.2003.822018 mixed potential integral equation (MPIE) in the spatial domain. Indeed, the relative overall CPU time saving arising from the use of DCIT is even more important in 2-D problems. In this latter case, the size of the matrices involved is not as large as in 3-D problems and, therefore, the relative computational weight of the few reaction entries is more significant in the total CPU time. For that reason, any refinement in the application of the DCIT to transmission-line problems is justified from the computational point-of-view. Thus, the DCIT was first adapted to analyze the bound regime of 2-D planar/nonplanar transmission lines in layered media [7]-[11]. The above 2-D studies were extended in [12] to also deal with the leaky regimes of printed lines. In spite of the progress reported in the aforementioned papers, there are still important questions concerning the application of the DCIT to 2-D problems that demand further discussions. This is the case, for example, of the appropriate choice of the straight-line paths to be used for sampling the spectral Green's functions in the frame of the two-levels scheme proposed in [6]. It can be observed that the sampling path used for 2-D structures [8]-[12] is actually the same as that proposed in [6] for 3-D problems. The choice of this sampling path was properly discussed in this latter work and is based on the convenience of sampling the spectral Green's functions in a region near the inverse integration path used in spectral-domain analysis (SDA) to obtain their spatial counterparts. However, it has been somewhat assumed that this suitable sampling path for 3-D problems is equally convenient for 2-D cases. This study will show that the suitable path for sampling the spectral Green's function in 2-D problems may not be that used in 3-D problems. In fact, the spatial functions are not obtained in 2-D SDA from their spectral counterparts by means of a Fourier-Bessel transform in the radial wavenumber $k_{\rho}$, but performing an inverse Fourier transform in the lateral wavenumber $k_{x}$ for a given fixed value of the longitudinal wavenumber $k_{z}$ with $k_{\rho}^{2}=k_{x}^{2}+k_{z}^{2}$. The new sampling paths proposed in this paper will provide superior accuracy for bound modes computation. Moreover, the requirement of different integration paths in the $k_{x}$ complex plane for surface- and space-leaky modes [13] has substantial consequences on the choice of the sampling path in the upper half-space vertical wavenumber $\left(k_{y 0}\right)$ complex plane. (It should be noted that this latter wavenumber is the proper variable to carry out the complex images expansion [4], [14].) A systematic study of the region covered by $k_{y 0}$ for the different values of the SDA integration path is carried out for each type of line modes in order 
to propose suitable sampling paths for bound, surface-leaky, and space-leaky modes.

It has been pointed out in the literature that, in general, it is very convenient to extract the poles of the spectral Green's functions associated with surface-wave (SW) modes of the background waveguide in both 3-D [1]-[4], [14] and 2-D problems [9], [10], [12]. Nevertheless, the use of the sampling paths proposed in this paper can make unnecessary pole extraction in the case of bound modes. There also seems to lack a detailed discussion about the suitability of the extraction of poles associated with improper modes of the background waveguide. Moreover, the extraction of these types of poles cannot be carried out by making use of the strategy used thus far in the literature to extract the pair of poles related to SW modes. In this paper, a new pole-extraction strategy is presented, which is able to deal with poles associated with $\mathrm{SW}$, as well as improper modes of the background waveguide. In addition, a systematic discussion on which poles must be extracted when searching for each type of line modes is carried out.

This paper is organized as follows. Section II will briefly discuss some aspects related to poles and asymptotic behavior extraction when the DCIT is employed to solve the MPIE for planar lines. Explicit expressions are given for the spatial counterparts of each term used in the treatment of the spectral Green's functions. In Section III, a thorough discussion on the sampling paths and the convenience of the pole extraction is carried out. This study is completed by diverse numerical results for bound as well as surface- and space-leaky modes. Finally, the DCIT is applied in Section IV to the analysis of covered 2-D planar structures. Numerical results for bound/leaky modes will show that the approximation given by the DCIT for the spectral Green's functions is sufficiently accurate leading, in general, to good agreement with SDA results.

\section{ANALYSIS}

\section{A. Formulation of the Problem}

The transmission line under study (see Fig. 1) consists of $N_{c}$ infinitely thin perfect conducting strips embedded in an $N$-layer planar substrate. All the layers are isotropic dielectrics of permittivity $\varepsilon_{i}(i=1, \ldots, N)$. The usual $\exp \left[-j\left(k_{z} z-\omega t\right)\right]$ phase factor will be assumed for all the fields and currents, with $k_{z}$ being the wavenumber along the longitudinal direction and $\omega$ being the angular frequency.

Following [9] and [15, formulation C], the MPIE for this problem can be written as

$$
\left.\begin{array}{r}
\hat{\mathbf{y}} \times \sum_{i=1}^{N_{c}}\left\{j \omega \int_{-\frac{w_{i}}{2}}^{\frac{w_{i}}{2}} G_{x x}^{A}\left(x_{j}, y_{j} \mid x^{\prime}, y_{i}^{\prime}\right) \mathbf{J}\left(x^{\prime}\right) d x^{\prime}+\left(\nabla_{t}-j k_{z} \hat{\mathbf{z}}\right)\right. \\
\left.\cdot \int_{\frac{-w_{i}}{2}}^{\frac{w_{i}}{2}} G_{\phi}\left(x_{j}, y_{j} \mid x^{\prime}, y_{i}^{\prime}\right) \frac{j}{\omega}\left(\nabla_{t}^{\prime}-j k_{z} \hat{\mathbf{z}}\right) \cdot \mathbf{J}\left(x^{\prime}\right) d x^{\prime}\right\} \\
=\mathbf{0}, \quad x_{j} \in\left(-\frac{w_{j}}{2}, \frac{w_{j}}{2}\right) ; j=1, \ldots, N_{c}
\end{array}\right\}
$$

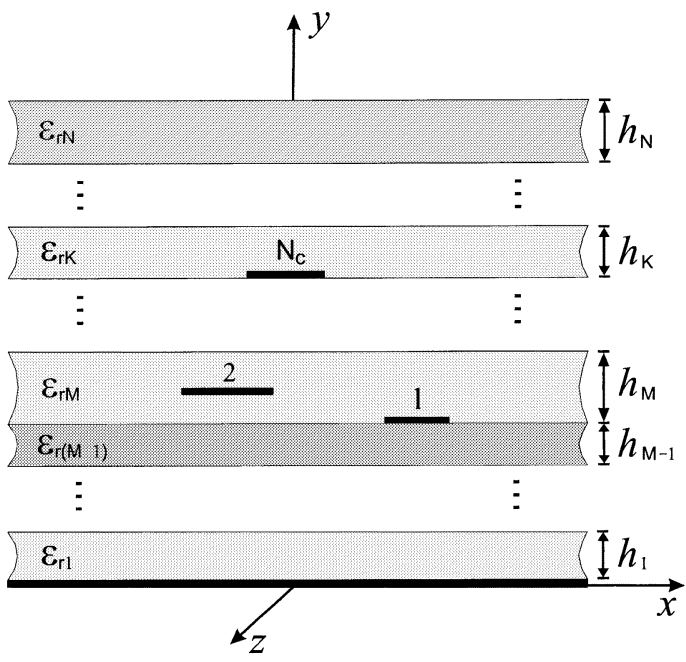

Fig. 1. Cross section of the multilayer and multiconductor transmission line under study.

where $w_{k}$ is the $k$ th strip width, $y_{k}$ is its vertical position, $\nabla_{t}$ is the transverse-to- $z$ nabla operator, $G_{x x}^{A}$ is the $\hat{\mathbf{x}} \hat{\mathbf{x}}$ component of the dyadic Green's function associated with the magnetic vector potential (A), $G_{\phi}$ is the Green's function associated with the scalar electric potential $(\phi)$, and $\mathbf{J}$ is the surface electric current density on the conducting strips. (Due to the choice of [15, formulation C], which implies $G_{x z}^{A}=G_{z x}^{A}=0$, and to the cylindrical symmetry of the background grounded layered waveguide, which causes $G_{z z}^{A}=G_{x x}^{A}$, only the $\hat{\mathbf{x}} \hat{\mathbf{x}}$ component of the dyadic Green's function for $\mathbf{A}$ is needed to describe, in general, the case of infinitely thin conductors [9].)

Since the kernels $G_{x x}^{A}$ and $G_{\phi}$ of the above eigenvalue equation for $k_{z}$ are not known in closed form, they are better computed from their spectral-domain counterparts

$$
\widetilde{G}\left(k_{x} ; y, y^{\prime}\right)=\int_{-\infty}^{\infty} G\left(x, y \mid x^{\prime}, y^{\prime}\right) e^{j k_{x}\left(x-x^{\prime}\right)} d x
$$

( $G$ stands for either $G_{x x}^{A}$ or $G_{\phi}$ ), which can be easily expressed in closed form using the transmission-line network analogous [15], [16]. Space-domain kernels are then obtained by performing the following inverse Fourier transform:

$$
G\left(\left|x-x^{\prime}\right| ; y, y^{\prime}\right)=\frac{1}{2 \pi} \int_{-\infty}^{\infty} \widetilde{G}\left(k_{x} ; y, y^{\prime}\right) e^{-j k_{x}\left(x-x^{\prime}\right)} d k_{x}
$$

Obtaining an accurate closed form for (3) for any type of mode is the final goal of this paper.

\section{B. Green's Functions in the Spectral Domain}

Basically, the DCIT [1], [2] is based on the possibility of finding an approximation of the spectral version of the kernel in (1) (or of a part of it) in terms of a finite sum of complex exponentials (the space-domain version of each exponential is known in closed form). Due to the cylindrical symmetry of the background waveguide (the waveguide in Fig. 1 without the 
metallic strips), $\widetilde{G}$ does not depend separately on $k_{x}$ and $k_{z}$, but on $k_{\rho}=\sqrt{k_{x}^{2}+k_{z}^{2}}$. Since the DCIT will only work properly when applied to analytic functions, before attempting the aforementioned expansion of $G\left(k_{\rho}\right)$, this function must be adequately treated and its analytic part identified. First, it should be reminded that the spectral Green's function has branch-point singularities at $k_{\rho}= \pm k_{0}$ [16], with $k_{0}=\omega \sqrt{\varepsilon_{0} \mu_{0}}$ being the free-space wavenumber. In addition, $\widetilde{G}_{\phi}\left(k_{\rho}\right)$ has one pair of poles corresponding to each background waveguide mode, whereas $\widetilde{G}_{x x}^{A}\left(k_{\rho}\right)$ has one pair of poles corresponding to each TE waveguide mode [15, formulation C]. It is also very convenient to extract the asymptotic behavior for large values of $k_{x}$ provided that a closed-form expression for its spatial counterpart can be found.

Relying on physical and/or mathematical arguments, it is clear that the branch points of the spectral Green's function can be removed if $\widetilde{G}\left(k_{\rho}\right)$ is written as a function of

$$
k_{y 0}=\sqrt{k_{0}^{2}-k_{x}^{2}-k_{z}^{2}}
$$

rather than in terms of $k_{\rho}\left(k_{y 0}\right.$ is the wavenumber along the $y$-direction in the upper half-space) since this transformation makes the spectral Green's function single valued in the $k_{y 0}$-plane. (It should be noticed that any other choice of the vertical wavenumber variable, such as using the vertical wavenumber inside an arbitrary layer of dielectric, would not be appropriate to reach the above goal). Moreover, the functions used in the DCIT are exponentials of $k_{y 0}$ and the quasi-static behavior must be written as a function of $k_{y 0}$ in order to be properly extracted [4], [9]. Concerning the extraction of poles, the $k_{y 0}$ variable naturally leads to a different strategy not having the fundamental limitations inherent to the extraction by pairs in the $k_{\rho}$-plane used thus far in the literature (these limitations will be commented upon and discussed further). For all the above reasons, the choice of $k_{y 0}$ as the working variable makes very natural and readily understandable the expansion in terms of complex exponentials and the extraction of both the poles and asymptotic behavior.

Next, it will be shown how to extract an arbitrary set of $N_{p}$ poles $\left\{k_{y 0, p}\right\}_{p=1, \ldots, N_{p}}$ in the $k_{y 0}$-plane. Since the poles do not appear in pairs in this plane, they can be extracted from the spectral Green's function by simply subtracting the following sum:

$$
\widetilde{G}^{P}\left(k_{y 0} ; y, y^{\prime}\right)=\sum_{p=1}^{N_{p}} \frac{R_{p}\left(y, y^{\prime}\right)}{k_{y 0}-k_{y 0, p}}
$$

where $R_{p}\left(y, y^{\prime}\right)$ is the residue of $\widetilde{G}\left(k_{y 0}\right)$ at $k_{y 0, p}$, which can be computed, using Cauchy's theorem, as

$$
R_{p}\left(y, y^{\prime}\right)=\frac{1}{2 \pi j} \oint_{C_{p}} \widetilde{G}^{P}\left(k_{y 0} ; y, y^{\prime}\right) d k_{y 0}
$$

with $C_{p}$ being a closed path (CP) around only the $k_{y 0, p}$ pole. Note that no restriction has been imposed to the nature of the above poles and, hence, $k_{y 0, p}$ can be any proper/improper pole of the spectral Green's function. Despite the convenience of the above strategy, the authors could not find a closed-form expression for the spatial counterpart of $\widetilde{G}^{P}$ (see Section II-C). Alternatively, the following $\widetilde{G}^{P}$ :

$$
\begin{aligned}
\widetilde{G}^{P}\left(k_{y 0} ; y, y^{\prime}\right) & =\sum_{p=1}^{N_{p}} \frac{-2 R_{p}\left(y, y^{\prime}\right) k_{y 0, p}}{k_{y 0}^{2}-k_{y 0, p}^{2}} \\
& =\sum_{p=1}^{N_{p}}\left[\frac{R_{p}\left(y, y^{\prime}\right)}{k_{y 0}-k_{y 0, p}}-\frac{R_{p}\left(y, y^{\prime}\right)}{k_{y 0}+k_{y 0, p}}\right]
\end{aligned}
$$

which does have a closed-form spatial counterpart, can be used to extract the poles. This strategy is very close to that used thus far in the literature for 2-D, as well as 3-D problems. However, it can be appreciated that this function introduces fictitious poles at $k_{y 0}=-k_{y 0, p}$. Furthermore, improper poles (poles with $\Im\left(k_{y 0, p}\right)>0$ ) cannot be extracted by using this alternative function (see Section II-C for an explanation). The importance of these facts when searching for bound/leaky modes will be discussed in Section III, where it is also considered the convenience of employing either (5) or (7).

Concerning the asymptotic behavior of the spectral Green's function (namely, the quasi-static limit in the spatial domain), the following expression is reported in [9]:

$$
\lim _{\left|k_{x}\right| \rightarrow \infty} \widetilde{G}\left(k_{y 0} ; y, y^{\prime}\right) \equiv \widetilde{G}^{0}\left(k_{y 0} ; y, y^{\prime}\right)=\frac{C\left(y, y^{\prime}\right)}{k_{y 0}}
$$

where $C$, for the scalar potential Green's function, is given by

$$
C_{\phi}=\left\{\begin{array}{l}
\frac{1}{j\left(\varepsilon_{n}+\varepsilon_{n+1}\right)}, \quad \text { for } y=y^{\prime}=h_{n} \\
\frac{1}{2 j \varepsilon_{n+1}}, \quad \text { for } h_{n}<y=y^{\prime}<h_{n+1} \\
0, \quad \text { for } y \neq y^{\prime}
\end{array}\right.
$$

and, for the vector potential,

$$
C_{x x}^{A}= \begin{cases}\frac{\mu_{0}}{2 j}, & \text { for } y=y^{\prime} \\ 0, & \text { for } y \neq y^{\prime} .\end{cases}
$$

If a pole extraction using (5) was carried out, $\widetilde{G}^{P}$ would introduce a new $k_{y 0}^{-1}$ asymptotic term, thus yielding the following expression for the total asymptotic behavior:

$$
\widetilde{G}^{\infty}\left(k_{y 0} ; y, y^{\prime}\right)=\frac{C\left(y, y^{\prime}\right)-\sum_{p=1}^{N_{p}} R_{p}\left(y, y^{\prime}\right)}{k_{y 0}} .
$$

Once both $\widetilde{G}^{P}$ and $\widetilde{G}^{\infty}$ have been subtracted from the spectral Green's function, the remaining function can be now properly approximated by a finite sum of complex images for given values of $y, y^{\prime}$ as follows:

$$
\begin{aligned}
\widetilde{G}\left(k_{y 0}\right)-\widetilde{G}^{P}\left(k_{y 0}\right)- & \widetilde{G}^{\infty}\left(k_{y 0}\right) \\
& \approx \frac{1}{k_{y 0}} \sum_{i=1}^{N_{e}} a_{i} e^{-j k_{y 0} \gamma_{i}} \equiv \widetilde{G}^{I}\left(k_{y 0}\right) .
\end{aligned}
$$

In practice, function $k_{y 0} \widetilde{G}^{I}\left(k_{y 0}\right)$ is approximated by a finite sum of exponentials using, for example, the generalized pencil of functions (GPoF) method [17]. Since this method requires a sampling of the function along a path, it will need a criterion 
to choose the regions of the complex $k_{y 0}$-plane in which the Green's functions must be sampled. Although this criterion has been discussed in [1], [2], and [6] for the 3-D problem, a similar discussion seems to be lacking in the 2-D frame. Section III will show that this criterion arises naturally and becomes rather clear when searching for proper, as well as improper/leaky modes by inspection of the mapping of the suitable SDA integration path onto the $k_{y 0}$-plane.

\section{Green's Functions in the Spatial Domain}

Although no closed-form expression was found for the contribution of $\widetilde{G}^{P}$ in (5) to the spatial Green's function, it has been possible to obtain a quasi-analytical expression. The use of asymptotic and complex plane integration techniques has provided an efficient algorithm with gives sufficient accuracy with low computational effort, as explained in the Appendix. On the other hand, (7) does have a direct closed-form spatial counterpart in the case of proper poles associated with SW modes of the background waveguide. Thus, assuming that only one SW pole is to be extracted, (7) can be rewritten as

$$
\begin{aligned}
\widetilde{G}^{P}\left(k_{y 0} ; y, y^{\prime}\right) & =\frac{2 R_{p}\left(y, y^{\prime}\right) k_{y 0, p}}{k_{x}^{2}-k_{x p}^{2}} \\
& =R_{p}\left(y, y^{\prime}\right) \frac{k_{y 0, p}}{k_{x p}}\left(\frac{1}{k_{x}-k_{x p}}-\frac{1}{k_{x}+k_{x p}}\right)
\end{aligned}
$$

where $k_{x p}=\sqrt{k_{0}^{2}-k_{z}^{2}-k_{y 0, p}}$ is the location of the pole in the $k_{x}$-plane (this function has a pair of poles at $k_{x}= \pm k_{x p}$ ). The spatial counterpart of (13) can be evaluated by a simple residue calculus for the case of bound, as well as surface-leaky (with respect to this SW mode) modes. Although this computation was already carried out in [12], it will be reproduced briefly here for completeness. It is well known in the frame of the SDA [13] that, for the case of bound modes, the integration path runs along the real axis of the $k_{x}$-plane and, hence, the inverse Fourier transform can be expressed in terms of the pole having $\Im\left(k_{x p}\right)<0$. Otherwise, if the mode is improper with respect to this SW mode, the integration path no longer lies on the real axis, but it detours around the pair of SW poles [13], thus taking the residue of that pole satisfying $\Im\left(k_{x p}\right)>0$. These considerations lead to the following expression for the spatial counterpart of (7):

$$
G^{P}\left(\left|x-x^{\prime}\right| ; y, y^{\prime}\right)=-j R_{p}\left(y, y^{\prime}\right) \frac{k_{y 0, p}}{k_{x p}} e^{-j k_{x p}\left|x-x^{\prime}\right|}
$$

where $k_{x p}$ is chosen with negative imaginary part if the mode is bound and with positive imaginary part if it is improper with respect to the SW waveguide mode.

Unfortunately, an important drawback of the above pole-extraction strategy is that poles associated with improper waveguide modes cannot be extracted. If such a pair of poles were extracted using (7), a nonexistent pair of poles associated with a fictitious proper waveguide mode would be unavoidably introduced. Note that similarly when a pair of proper poles are extracted out, a nonexistent pair of improper poles are also introduced. Nevertheless, this latter case does not pose special drawbacks because these fictitious improper poles lie on the im-

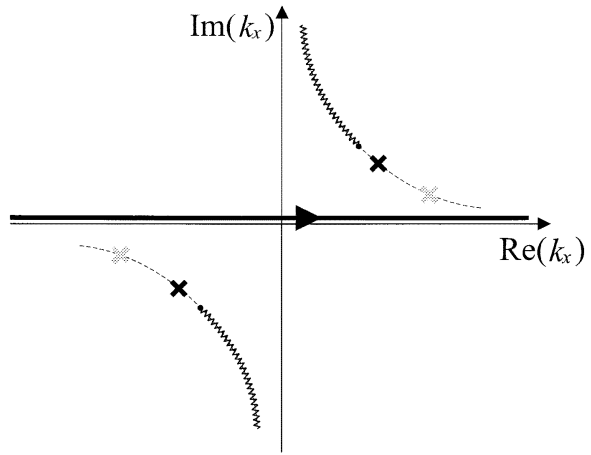

(a)

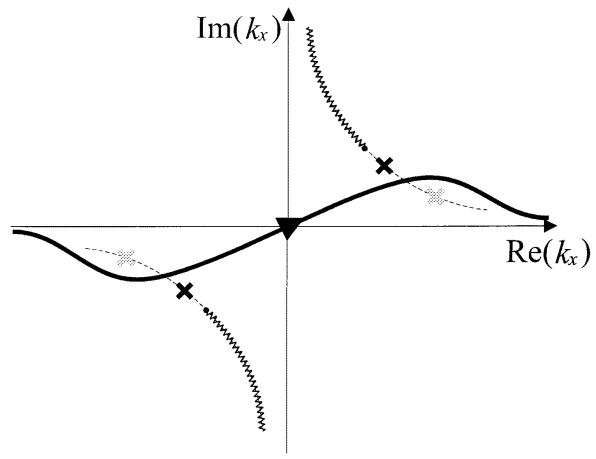

(b)

Fig. 2. Two equivalent SDA integration paths when searching for bound modes.

proper sheet in the $k_{x}$-plane, whereas the integration path lies on the proper sheet. On the contrary, the fictitious pair of proper poles would account for a new proper SW mode that has an important effect on the behavior of the Green's function on the proper sheet. In consequence, it should be decided how its corresponding poles must contribute to the inverse Fourier transform. Nevertheless, any choice about these poles will be meaningless because this mode does not actually exist and cannot play any mathematical/physical role in our problem. To illustrate the above discussion, it will be considered a case where a pair of poles associated with an improper real mode are extracted and a bound mode is searched for. For simplicity, it will be assumed that only the $\mathrm{TM}_{0}-\mathrm{SW}$ mode is above cutoff at the working frequency. This situation is depicted in Fig. 2(a), which shows the Sommerfeld branch cuts, integration path along the real axis, pair of $\mathrm{TM}_{0}-\mathrm{SW}$ poles (black crosses), and also the pair of poles associated with the fictitious mode (grey crosses) that appear on the proper sheet of the Riemann surface. (It is convenient to recall that these latter poles are not present in the spectral Green's function.) Looking now at Fig. 2(b), the integration path shown here should be completely equivalent to that in Fig. 2(a) since any of them can be continuously deformed to match the other one without capturing any actual singularity of the spectral Green's function. However, it is apparent that the contribution of $\widetilde{G}^{P}$ to the spatial-domain Green's function is not the same when using the integration paths shown in Fig. 2(a) and (b). This ambiguity in $G^{P}$, which is caused by the introduction of a nonexistent pair of proper poles, indicates that the extraction of poles related to improper waveguide modes by means of (7) is fundamentally unfeasible. 
Finally, the asymptotic $\widetilde{G}^{\infty}$ and regular $\widetilde{G}^{I}$ contributions to the spatial Green's function can be obtained in closed form after using identity [9, eq. (11)], which can be rewritten as

$$
\begin{aligned}
\frac{1}{2 \pi} \int_{-\infty}^{\infty} \frac{e^{-j k_{y 0} \xi}}{j k_{y 0}} e^{-j k_{x}\left(x-x^{\prime}\right)} d k_{x} & \\
= & \frac{1}{\pi} K_{0}\left(j k_{x 0} \sqrt{\left(x-x^{\prime}\right)^{2}+\xi^{2}}\right)
\end{aligned}
$$

where $K_{0}(\cdot)$ is the modified Bessel function of the second kind and order zero and $k_{x 0}^{2}=k_{0}^{2}-k_{z}^{2}$. Thus, by comparison of (15) with (11) and (12), the spatial counterparts of $\widetilde{G}^{\infty}$ and $\widetilde{G}^{I}$ are found to be

$$
\begin{aligned}
& G^{\infty}\left(\left|x-x^{\prime}\right| ; y, y^{\prime}\right) \\
& =\frac{j}{\pi}\left[C\left(y, y^{\prime}\right)-\sum_{p=1}^{N_{p}} R_{p}\left(y, y^{\prime}\right)\right] K_{0}\left(j k_{x 0}\left|x-x^{\prime}\right|\right) \\
& G^{I}\left(\left|x-x^{\prime}\right| ; y, y^{\prime}\right) \\
& \quad=\frac{j}{\pi} \sum_{i=1}^{N_{e}} a_{i}\left(y, y^{\prime}\right) K_{0}\left(j k_{x 0} \sqrt{\left(x-x^{\prime}\right)^{2}+\gamma_{i}^{2}\left(y, y^{\prime}\right)}\right) .
\end{aligned}
$$

As was pointed in the above section, the sum term in (16) is present only if (5) has been used to extract poles. Note that, due to this sum term, the spatial Green's function seems to present a nonphysical logarithmic singularity for $x=x^{\prime}$ when $y \neq y^{\prime}$. This singularity is analytically cancelled by the logarithmic singularity of the corresponding exponential integral functions that appear in the spatial counterpart of $\widetilde{G}^{P}$ (see the Appendix), which means that only the correct quasi-static singularity is present in the spatial Green's functions. In (16) and (17), $k_{x 0}$ must be chosen with negative imaginary part if the mode is bound or surface leaky and with positive imaginary part for space-leaky modes [12].

\section{Discussion on SAmpling Paths AND Pole Extraction}

In [1]-[4], a single straight-line sampling path was used to approximate the spectral Green's functions (one-level approach) by means of the DCIT. A two-level approach based on a doublescale sampling of a single straight line was then proposed by Kipp and Chan in [5]. Later, Aksun showed in [6] that a twolevel approach using a sampling path now composed of two straight lines was very convenient to overcome some difficulties associated with the robustness and efficiency of the former approach. This latter scheme showed a very good performance when used in the subsequent works devoted to the specific application of the DCIT to solve 2-D planar and nonplanar transmission-line problems [8]-[12]. This approach is then adopted in this study and our discussion will focus on how to choose suitably the two straight lines of the sampling path when searching for the different types of line modes. This discussion will be based on the study of the regions of the $k_{y 0}$-plane explored by the $k_{x}$-plane integration paths used in the SDA formulation to carry out the inverse transform (3) for each type of modes.

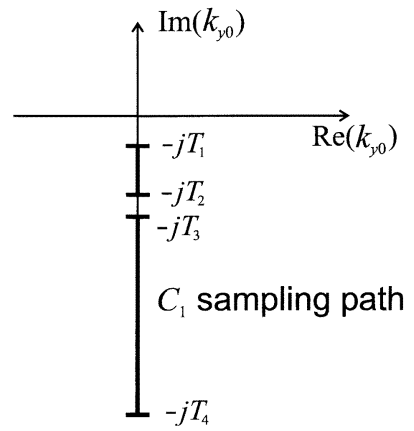

(a)

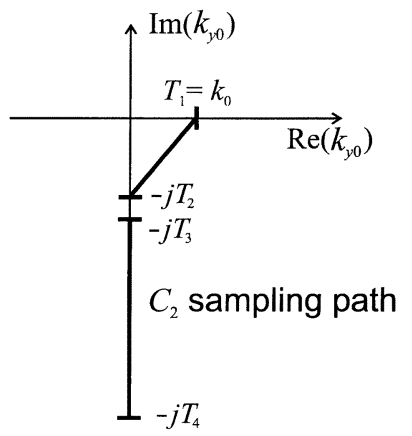

(b)

Fig. 3. (a) Two-level sampling path suitable for proper modes proposed in this paper. (b) The sampling path used in [8]-[12].

\section{A. Bound Modes}

Bound (proper) modes are obtained in SDA by performing the integral in (3) along the real axis on the proper sheet of the $k_{x}$-plane $\left(-\infty<k_{x}<\infty\right)$. Taking into account (4) and that, for these modes, $k_{z}$ is real and greater than the propagation constant of any SW mode at the working frequency, the mapping of the above integration path onto the complex $k_{y 0}$-plane corresponds to values of $k_{y 0}$ lying on the negative imaginary axis from $-j \infty$ to $-j\left|k_{x 0}\right|$. The upper limit $-j\left|k_{x 0}\right|$ lies near and below the $k_{y 0}$ value associated with the $\mathrm{TM}_{0}-\mathrm{SW}$ wavenumber. Thus, the appropriate two-level sampling path should have both sampling segments on the negative imaginary axis. Such a path, which will be referred to as $C_{1}$, is illustrated in Fig. 3(a), whereas Fig. 3(b) shows the path $C_{2}$ used in [8]-[12] following [6]. Since the $k_{y 0}$ poles associated with SW modes lie in the portion of the $k_{y 0}$ negative imaginary axis where $-\sqrt{k_{\max }-k_{0}}<\Im\left(k_{y 0}\right)<0$, with $k_{\max }=\max \left\{\omega \sqrt{\varepsilon_{i} \mu_{0}}\right\}_{i=1, \ldots, N}$, a suitable choice for the starting point of the upper sampling segment (the fine sampling region) is $T_{1} \gtrsim \sqrt{k_{\max }-k_{0}}$. Values of $T_{4}$ and $T_{3}$ can be chosen to be $100 k_{0}$ and $10 k_{0}$ (long-term sampling region), respectively, and $T_{2}$ can be taken about $8 k_{0}-9 k_{0}$. The values given above have been tested in very different situations (a wide range of frequencies, number of layers and/or strips, permittivities, etc.) and they have always given a good performance. The gap between both sampling segments is convenient because, according to the two-level sampling scheme, the function has been already approximated in $-j T_{3}$ when the approximation in the upper sampling segment is carried out. If $T_{2}$ was chosen to be equal to $T_{3}$, the function to be approximated in this second 

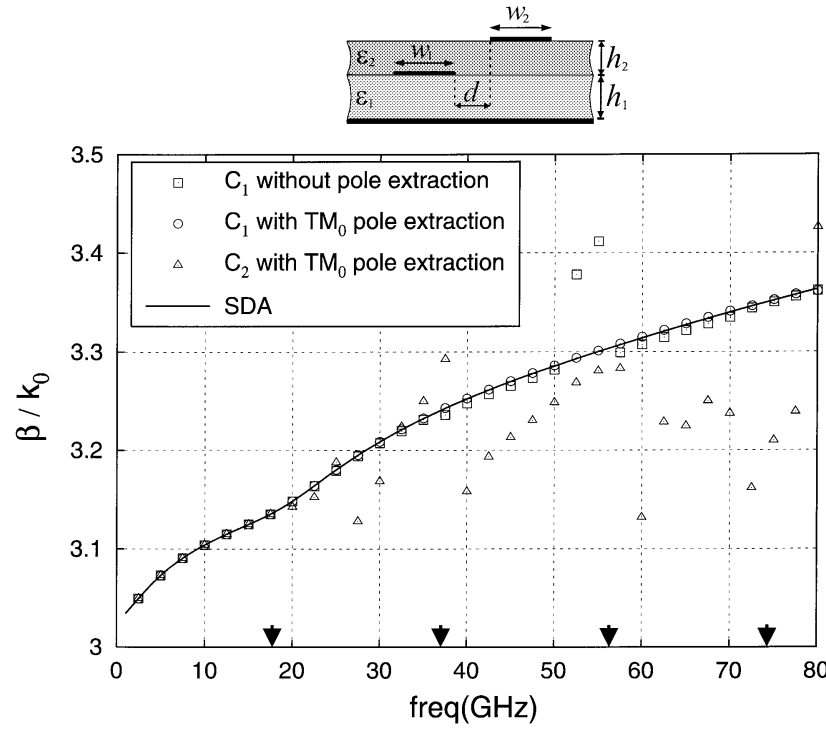

Fig. 4. Normalized phase constant for the structure shown with $\varepsilon_{1}=9.8 \varepsilon_{0}$, $\varepsilon_{2}=12.5 \varepsilon_{0}, h_{1}=h_{2}=0.635 \mathrm{~mm}, w_{1}=3 \mathrm{~mm}, w_{2}=2 \mathrm{~mm}$, and $d=2.5 \mathrm{~mm}$.

segment would present an abrupt decay at $k_{y 0}=-j T_{3}$ that may lead to an inaccurate second-level approximation.

For 2-D problems, in [9], the convenience of extracting all SW poles, similar to what happens for 3-D structures, was pointed out. The reason argued was that complex exponentials are not able to accurately reproduce the behavior associated with these poles in the spectral domain. A careful analysis will show that this is true only if path $C_{2}$ is used for sampling and that the spectral Green's function can be very accurately approximated along the sampling path $C_{1}$ without extracting the SW poles. Moreover, the required number of complex exponentials turns out to be quite low when $C_{1}$ is employed. Certainly, this strategy could fail when the $\mathrm{TM}_{0}$ pole lies very close to $-j T_{1}$. Nevertheless, our experience suggests that this drawback is only important for frequencies at which at least three SW modes are above cutoff. Even in such a case, this problem can be overcome by extracting only the $\mathrm{TM}_{0}$ pole. This extraction can be carried out using (7) since the added nonexistent pole will lie on the positive imaginary axis, far away from the sampling path and, therefore, it is not expected to affect the quality of the approximation. Evidently, the problem reappears when the first higher $\mathrm{SW}$ mode approaches $-j T_{1}$, although it only occurs when six or more SW modes are above cutoff, a situation not very common in practice. When the traditional path $C_{2}$ is employed, all the poles must be extracted in order to assure that the possible "good" approximation given by the GPoF in the upper segment of $C_{2}$ is still accurate on the upper segment of $C_{1}$, precisely over the region of interest. Since this latter region of the $k_{y 0}$-plane is part of the actual region for which the Green's function must be accurately computed, any robust strategy must ensure the approximation within this portion of the $k_{y 0}$-plane to be accurate enough.

The above ideas are illustrated in Fig. 4, which shows the computed values obtained for the normalized phase constant $\beta / k_{0}$ of the fundamental quasi-odd mode of a two-layer twostrip line. The values of the parameters of the $C_{1}$ and $C_{2}$ sam-
TABLE I

RESUlTS OBTAINED FOR THE FUNDAMENTAL MODE OF A SingLE-STRIP SINGLE-LAYER TRANSMISSION LINE WITH $\varepsilon=9.8 \varepsilon_{0}, h=0.635 \mathrm{~mm}$ $w=3 \mathrm{~mm}$, AND Freq $=32 \mathrm{GHz}$. SDA RESULT: $k_{z}=3.0342 k_{0}$

\begin{tabular}{c|c|c|c|c|c}
\hline \multicolumn{2}{l|}{} & \multicolumn{2}{c|}{$C_{1}$ sampling path } & \multicolumn{2}{c}{$C_{2}$ sampling path } \\
\hline$N_{\epsilon 1}$ & $N_{\epsilon 2}$ & $\Re\left(k_{z}\right) / k_{0}$ & $\Im\left(k_{z}\right) / k_{0}$ & $\Re\left(k_{z}\right) / k_{0}$ & $\Im\left(k_{z}\right) / k_{0}$ \\
\hline \hline 1 & 2 & 3.0342 & $\sim 10^{-16}$ & 3.0462 & 0.0116 \\
\hline 1 & 3 & 3.0342 & $\sim 10^{-16}$ & 3.0338 & $\sim 10^{-5}$ \\
\hline 2 & 3 & 3.0342 & $\sim 10^{-16}$ & 3.0319 & $\sim 10^{-4}$ \\
\hline 2 & 4 & 3.0342 & $\sim 10^{-15}$ & 3.0266 & $-8 \times 10^{-4}$ \\
\hline 3 & 4 & 3.0342 & $\sim 10^{-16}$ & 3.0340 & $5 \times 10^{-4}$ \\
\hline 3 & 5 & 3.0342 & $\sim 10^{-14}$ & 3.0341 & $\sim 10^{-6}$ \\
\hline 4 & 5 & 3.0342 & $\sim 10^{-14}$ & 3.0342 & $\sim 10^{-6}$ \\
\hline
\end{tabular}

pling paths used in this calculations were $T_{4}=100 k_{0}, T_{3}=$ $10 k_{0}, T_{2}=8 k_{0}$, and $T_{1}=3.4 k_{0}$, the total number of exponentials used to approximate the spectral Green's function up to $70 \mathrm{GHz}$ is seven - three for the first sampling segment and four for the second one-and one more exponential is added to approximate the second sampling segment above $70 \mathrm{GHz}$. The cutoff frequency of the third SW mode of the background waveguide is approximately $37 \mathrm{GHz}$ (the arrows on the horizontal axis indicate the cutoff frequencies of the higher order SW modes). For frequencies below $37 \mathrm{GHz}$, Fig. 4 shows an excellent agreement between the data obtained using $C_{1}$ without extracting any SW pole and the SDA analysis (which can be considered exact for our purpose). Beyond $37 \mathrm{GHz}$, the approximation given by $C_{1}$ without pole extraction deteriorates sensibly and can become unstable in some instances, as indicated by the two spurious values obtained for 52.5 and $55 \mathrm{GHz}$. On the other hand, the approximation involving $C_{1}$ and the extraction of just the $\mathrm{TM}_{0}-\mathrm{SW}$ poles gives an excellent agreement with the SDA results in all the high-frequency range (note that, at $80 \mathrm{GHz}$, five SW modes are above cutoff). On the contrary, the results given by $C_{2}$ when the $\mathrm{TM}_{0}-\mathrm{SW}$ mode is extracted out are reliable only for frequencies below the cutoff frequency of the first higher SW mode, thus showing that all the poles should be extracted when this path is used. To give an idea of the computational efficiency of the present proposal, the CPU time employed on average to compute each value represented in Fig. 4 by means of a highly optimized SDA algorithm is at least three times longer than that required by the proposed algorithm on the same platform.

As a further proof of the suitability of this strategy, Table I shows the results obtained for the fundamental mode of a microstrip line using $C_{1}$ without pole extraction and $C_{2}$ with pole extraction, respectively. It has been chosen $T_{4}=100 k_{0}, T_{3}=$ $10 k_{0}, T_{2}=8 k_{0}$, and $T_{1}=3 k_{0} . N_{e 1}$ and $N_{e 2}$ are, respectively, the number of exponentials used in the first- and second-level approximation. The SDA result for the propagation constant of the fundamental mode of this microstrip line is $k_{z}=3.0342 k_{0}$. It can be observed that the use of $C_{1}$ makes the algorithm more robust in the sense that, although no SW pole extraction has been carried out, a lower number of complex exponentials is needed to give more accurate results. In fact, note that the relative error obtained when employing $C_{1}$ is still below $10^{-5}$ with 


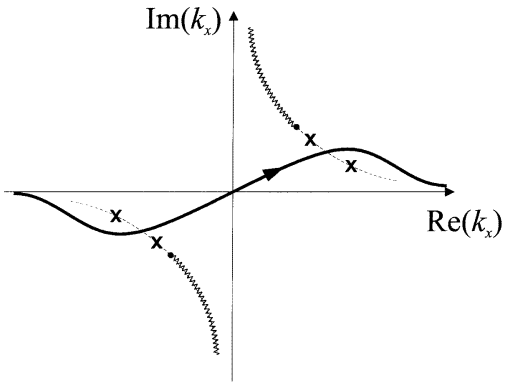

(a)

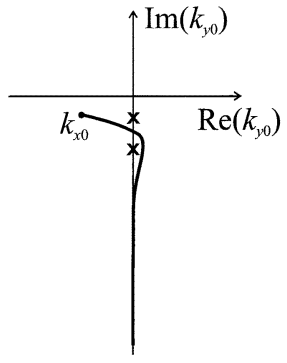

(b)

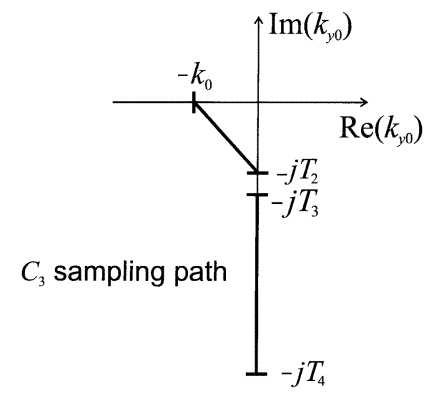

(c)

Fig. 5. (a) Integration path in the complex $k_{x}$-plane for surface leaky modes Two SW modes of the background waveguide are above cutoff although only the dominant mode is detoured around. (b) Mapping of the above integration path onto the complex $k_{y 0}$-plane. (c) Proposed fixed sampling path.

only three exponentials, whereas the results obtained by sampling along $C_{2}$ show that at least nine exponentials are required to give a comparable precision. (No more than five significant digits have been achieved in the computation of the real part of $k_{z}$ by means of DCIT for the parameters used in the table.) The structure in Table I has also been analyzed in [18], where the propagation constants for the fundamental and first two higher order modes are provided for several frequencies. Those results, which are particularly accurate, have been reproduced within, at least, four significant digits by using the $C_{1}$ sampling path proposed in this paper without extraction of poles. This strategy is, in the worst case, at least as efficient as using the $C_{2}$ path in [9] (which requires pole extraction).

\section{B. Surface-Leaky Modes}

It is well known that the $k_{x}$-plane integration path used in SDA to compute surface-leaky modes does not lie along the real axis, but it detours around the poles that contribute to the lateral radiation, although still lying on the proper Riemann sheet [13]. A typical integration path of this type is illustrated in Fig. 5(a) for the simplest case when lateral radiation takes place in the form of the dominant $\mathrm{TM}_{0}-\mathrm{SW}$ mode of the background waveguide although another SW mode is also above cutoff. The excursion of the integration path in the complex $k_{x}$-plane outside the real axis is mapped onto the $k_{y 0}$-plane as an excursion of $k_{y 0}$ in the third quadrant starting at the point $k_{y 0}\left(k_{x}=0\right)=k_{x 0}=\sqrt{k_{0}^{2}-k_{z}^{2}}$, as shown in Fig. 5(b).

According to this, it is apparent the convenience of choosing a sampling path whose upper sampling segment runs into the third quadrant starting at $k_{y 0}=k_{x 0}$. Such a path will then depend on $k_{z}$ and, therefore, it would imply a different sampling path and a new GPoF approximation for each value of $k_{z}$ during the searching process of the line propagation constant. It is then preferred a sampling path like $C_{3}$ in Fig. 5(c), which can be fixed for the whole process, although it will oblige us to extract all the SW modes for the same reasons adduced for the case of bound modes using $C_{2}$. Clearly, the poles associated with the lateral radiation should be taken with $\Im\left(k_{x p}\right)>0$ and the remaining ones with $\Im\left(k_{x p}\right)<0$. The poles can be safely extracted making use of (7) according to the rationale explained in Section III-A.

If a sampling path like $C_{2}$ was used, all the $\mathrm{SW}$ poles should be also extracted out, although it would be advisable to carry out this extraction by means of (5). This is because the nonexistent poles introduced by (7) may, in general, cause the approximation obtained along the upper sampling segment of $C_{2}$ to not give accurate results in the region of interest in the third quadrant of the complex $k_{y 0}$-plane. For the same reasons, if some real improper mode is not far enough from the $k_{y 0}$-plane origin, it must also be extracted. Of course, this latter extraction must be done using (5) since (7) is not able to deal with improper poles. To illustrate these ideas, Table II shows the results obtained for the propagation constant of a surface-leaky mode of a microstrip line by extracting the SW poles using either (7) or (5). Both $C_{2}$ and $C_{3}$ are delimited by $T_{4}=100 k_{0}, T_{3}=10 k_{0}$, and $T_{2}=9 k_{0}$. It can be observed in Table II(A) that the results obtained by using the sampling path $C_{3}$ are very accurate and convergent, while those obtained by means of $C_{2}$ are quite poor because of the nonexistent poles introduced by the pole extraction and the existence of an improper real mode at $k_{y 0} / k_{0}=j 1.1943$. In Table II(B), this improper real pole has been also extracted. Note that, in this table, the results obtained with $C_{3}$ are as accurate as those in Table II(A), but the values obtained by sampling on $C_{2}$ are now more accurate as a consequence of a suitable extraction of both the improper real and SW pole. Apart from the improvement of the $C_{2}$ results with respect to those in Table II(A), note that the values obtained by means of $C_{3}$ keep on being more accurate and convergent.

It should be pointed out that the above results correspond to values of the starting point of the sampling path $k_{x 0}$ located near the negative real axis $\left(k_{x 0} / k_{0}=-1.34-j 0.15\right.$ for the SDA $k_{z}$ value). For values of $k_{x 0}$ near the imaginary axis, a fixed sampling path like that depicted in Fig. 6, which will be referenced as $C_{4}$, becomes more suitable. In such a case, it is apparent that the robustness of the approximation given by the sampling path $C_{2}$ is less affected by either the presence of the improper real pole or the false poles introduced because of the use of (7). Thus, the results are accurate enough whether $C_{4}$ or $C_{2}$ is used, but as far as $C_{4}$ samples the spectral Green's function in the zone of interest, this latter sampling path is preferable. The results reported in Table II were chosen with a $k_{x 0}$ value near the real axis with the aim of highlighting the advantages of the new pole extraction strategy and sampling paths presented in this study.

\section{Space-Leaky Modes}

Spatial leaky modes can be obtained using the DCIT just by choosing $\Im\left(k_{x 0}\right)>0$, which causes fields to grow in the vertical direction [12]. For the mode to be physically valid (i.e., for it to be excited by a practical source), $\Im\left(k_{x p}\right)$ must be chosen positive for all the SW modes of the background waveguide [13]. 
TABLE II

Results ObTained For a Surface LEAKy Mode of A Single Microstrip LINE WITH $\varepsilon=9.8 \varepsilon_{0}, h=0.635 \mathrm{~mm}, w=3 \mathrm{~mm}$, AND Freq $=32 \mathrm{GHz}$. The VALUe OBTAINED BY SDA Is $k_{z} / k_{0}=0.2274-j 0.9081$

\begin{tabular}{c|c|c|c|c|c}
\hline \multicolumn{6}{c}{$(\mathrm{A}):$ SW poles extracted using (7) } \\
\hline \multicolumn{2}{l|}{} & \multicolumn{2}{c}{$C_{3}$ sampling path } & \multicolumn{2}{c}{$C_{2}$ sampling path } \\
\hline$N_{e 1}$ & $N_{e 2}$ & $\Re\left(k_{z}\right) / k_{0}$ & $\Im\left(k_{z}\right) / k_{0}$ & $\Re\left(k_{z}\right) / k_{0}$ & $\Im\left(k_{z}\right) / k_{0}$ \\
\hline 1 & 2 & 0.2289 & -0.9074 & 0.2897 & -0.9604 \\
\hline 1 & 3 & 0.2277 & -0.9081 & 0.2534 & -0.8336 \\
\hline 2 & 3 & 0.2274 & -0.9080 & 0.2562 & -0.8398 \\
\hline 2 & 4 & 0.2268 & -0.9090 & 0.1780 & -0.8676 \\
\hline 3 & 4 & 0.2273 & -0.9085 & 0.1685 & -0.8877 \\
\hline 3 & 5 & 0.2273 & -0.9084 & 0.2311 & -0.9290 \\
\hline 4 & 5 & 0.2274 & -0.9083 & 0.2353 & -0.9270 \\
\hline 4 & 6 & 0.2274 & -0.9083 & 0.2379 & -0.9045 \\
\hline 5 & 6 & 0.2274 & -0.9083 & 0.2341 & -0.9009 \\
\hline
\end{tabular}

(B): SW poles extracted using (5)

\begin{tabular}{c|c|c|c|c|c}
\hline \multicolumn{2}{l|}{} & \multicolumn{2}{c|}{$C_{3}$ sampling path } & \multicolumn{2}{c}{$C_{2}$ sampling path } \\
\hline$N_{e 1}$ & $N_{e 2}$ & $\Re\left(k_{z}\right) / k_{0}$ & $\Im\left(k_{z}\right) / k_{0}$ & $\Re\left(k_{z}\right) / k_{0}$ & $\Im\left(k_{z}\right) / k_{0}$ \\
\hline \hline 1 & 2 & 0.2264 & -0.9098 & 0.2058 & -0.9295 \\
\hline 1 & 3 & 0.2285 & -0.9094 & 0.2380 & -0.9175 \\
\hline 2 & 3 & 0.2272 & -0.9078 & 0.2226 & -0.9179 \\
\hline 2 & 4 & 0.2260 & -0.9085 & 0.2224 & -0.9090 \\
\hline 3 & 4 & 0.2271 & -0.9084 & 0.2233 & -0.9091 \\
\hline 3 & 5 & 0.2271 & -0.9084 & 0.2265 & -0.9080 \\
\hline 4 & 5 & 0.2272 & -0.9083 & 0.2267 & -0.9080 \\
\hline 4 & 6 & 0.2272 & -0.9083 & 0.2265 & -0.9083 \\
\hline 5 & 6 & 0.2273 & -0.9082 & 0.2268 & -0.9083 \\
\hline
\end{tabular}

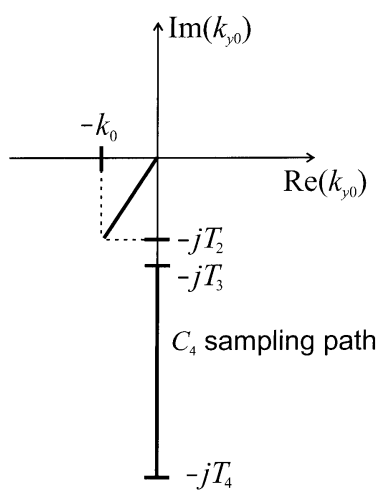

Fig. 6. $C_{4}$ sampling path suitable for values of $k_{x 0}$ near the imaginary axis.

In the case of space-leaky modes, the SDA $k_{x}$-plane integration path detours around the branch points crossing the Sommerfeld branch cuts and, therefore, lying partially on the improper sheet [13], as shown in Fig. 7(a). The fact that part of

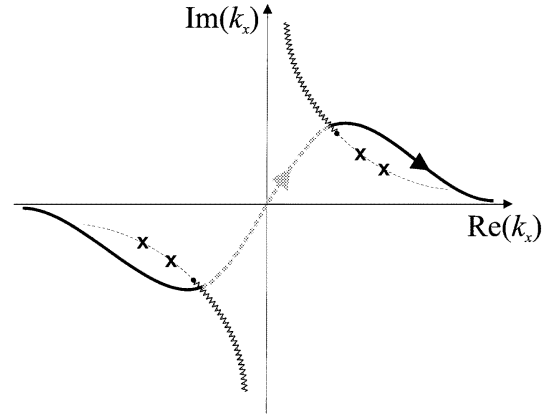

(a)

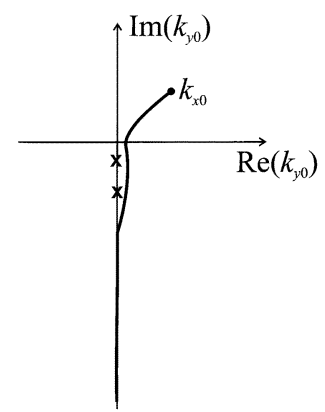

(b)

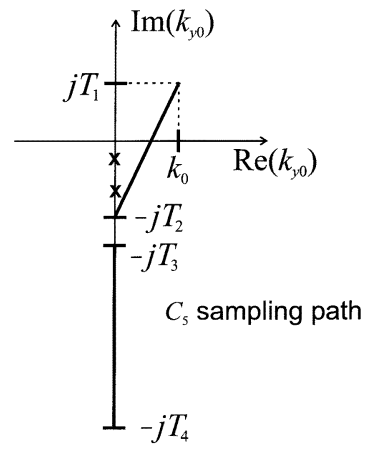

(c)

Fig. 7. (a) Integration path for spatial leaky modes. (b) Mapping of the integration path onto the $k_{y 0}$-plane. (c) Suitable $k_{y 0}$-plane sampling path.

the integration path runs along the improper sheet of the complex $k_{x}$-plane causes the corresponding mapped values in the $k_{y 0}$-plane to be located on the first quadrant [see Fig. 7(b)]. The values of $k_{y 0}$ corresponding to the proper part of the integration path will lie near the negative imaginary axis of the $k_{y 0}$-plane. As a consequence, a suitable sampling path could be that shown in Fig. 3(b), but with the starting point located in the $\Im\left(k_{y 0}\right)>0$ zone to allow for the second-level step of the approximation to explore the first quadrant. Such a path is depicted in Fig. 7(c) and will be denoted as $C_{5}$. Following the arguments given in previous sections, when $C_{5}$ is used, all the $\mathrm{SW}$ poles must be extracted by means of (5) to assure accurate results. However, our experience suggests that, whichever $C_{2}$ or $C_{5}$ was used, it is the extraction of poles associated to leaky waves (LWs) of the background waveguide what actually gives accurate results in a wide variety of situations. Since LW poles lie on the first quadrant of the $k_{y 0}$-plane, they may interpose between $C_{2}$ and the $k_{y 0}$-plane zone involved in the inverse transform or they may lie in the near vicinity of $C_{5}$ for a given frequency range. As a consequence, the second-level approximation of the spectral Green's function may be either not extendable to the region of interest when using $C_{2}$ or simply poor and unstable when the sampling path is $C_{5}$. It is then very convenient to extract those LW poles lying on the low-value range of the $k_{y 0}$-plane first quadrant. As a heuristic criterion, it has been found that the LW modes with $\left|k_{y 0, p}\right|<1.5 k_{0}$ must be extracted in order to assure proper results when either $C_{5}$ or $C_{2}$ is used to sample the spectral Green's function.

As a numerical illustration of the above ideas, consider the two-layer microstrip line whose parameters are give in Table III. At $55 \mathrm{GHz}$, the background waveguide presents a TM-LW mode 
TABLE III

RESUlts OBtained FOR A SPACE-LEAKy MODE OF A Two-LAyER MicrostriP LiNE WITH $\varepsilon_{1}=9.8 \varepsilon_{0}, \varepsilon_{2}=12.5 \varepsilon_{0}, h_{1}=h_{2}=0.635 \mathrm{~mm}$, $w=3 \mathrm{~mm}$, AND Freq $=55 \mathrm{GHz}$. THE CORRESPONDING SDA VALUE IS $k_{z} / k_{0}=(1.027-j 0.058)$

\begin{tabular}{c|c|c|c|c|c}
\hline \multicolumn{2}{l|}{} & \multicolumn{2}{c|}{$C_{5}$ sampling path } & \multicolumn{2}{c}{$C_{2}$ sampling path } \\
\hline$N_{e 1}$ & $N_{e 2}$ & $\Re\left(k_{z}\right) / k_{0}$ & $\Im\left(k_{z}\right) / k_{0}$ & $\Re\left(k_{z}\right) / k_{0}$ & $\Im\left(k_{z}\right) / k_{0}$ \\
\hline \hline 2 & 3 & 1.008 & -0.036 & - & - \\
\hline 2 & 4 & 1.038 & -0.057 & 1.044 & -0.044 \\
\hline 3 & 4 & 1.024 & -0.055 & 1.049 & -0.046 \\
\hline 3 & 5 & 1.024 & -0.058 & 1.031 & -0.058 \\
\hline 4 & 5 & 1.026 & -0.057 & 1.029 & -0.060 \\
\hline 4 & 6 & 1.026 & -0.056 & 1.030 & -0.060 \\
\hline 5 & 6 & 1.026 & -0.057 & 1.028 & -0.059 \\
\hline 5 & 7 & 1.027 & -0.058 & 1.027 & -0.058 \\
\hline 6 & 7 & 1.027 & -0.058 & 1.028 & -0.059 \\
\hline
\end{tabular}

at $k_{y 0} / k_{0}=1.0761+j 0.4882$ and a TE-LW mode at $k_{y 0} / k_{0}=$ $0.3269+j 0.6439$. When the propagation constant is computed using a $C_{2}$-like sampling path, no mode is found near the SDA value unless the LW poles were previously extracted. On the other hand, if the sampling path is $C_{5}$, but without $\mathrm{LW}$ poles extraction, values with less than $1 \%$ error are achieved for $N_{e 1}$ between 2-6 and $N_{e 2}=6$ and no mode is found near the SDA value for other ranges of $N_{e 1}$ and $N_{e 2}$. These facts indicate that although $C_{5}$ is more suitable than $C_{2}$, the approximation of the spectral Green's function is unstable when using $C_{5}$ and not valid for $C_{2}$ unless the LW poles are extracted. Thus, Table III shows the results obtained by using $C_{5}$ and $C_{2}$ with $T_{4}=100 k_{0}, T_{3}=10 k_{0}, T_{2}=9 k_{0}$, and $T_{1}=k_{0}$ when the aforementioned LW poles, as well as the three SW poles are extracted using (5). As no LW pole is now distorting the second-level approximation when using $C_{5}$, the results obtained for this sampling path are in very good agreement with SDA. Similarly, since no LW pole is now between $C_{2}$ and the region involved in the inverse transform, the propagation constant computed by using $C_{2}$ converges properly to the SDA value. This convergence is slightly faster when $C_{5}$ is used, which indicates that this latter sampling path is more appropriate.

\section{COVERED StRUCTURES}

This section will analyze the possible extension of the DCIT, previously developed for grounded lines, to the case of covered structures (namely, structures having a top ground plane). It has been widely argued that the good performance of the DCIT to give closed-form expressions for the spatial Green's function of a horizontal electric dipole in 3-D multilayered structures relies on the convenience of a field expansion in the upper half-space in terms of spherical waves [14]. Although this assumption is no longer strictly valid for covered structures and, therefore, it could be somewhat expected for the DCIT to work properly only for open structures, this technique has also been applied in [19] to deal with covered 3-D structures. This study showed that,
TABLE IV

SDA AND DCIT RESUlTS FOR THE PROPAGATION CONSTANT OF THE Fundamental Mode IN THE STRIPLINE SHOWN IN THE INSET OF Fig. 8(a) WITH $\varepsilon_{1}=10 \varepsilon_{0}, \varepsilon_{2}=\varepsilon_{0}, h_{1}=1 \mathrm{~mm}, h_{2}=0.5 \mathrm{~mm}$, AND $w=3 \mathrm{~mm}$

\begin{tabular}{c|c|c|c}
\hline \multicolumn{2}{l|}{} & \multicolumn{2}{c}{ DCIT } \\
\hline Freq $(\mathrm{GHz})$ & SDA & $\Re\left(k_{z}\right) / k_{0}$ & $\Im\left(k_{z}\right) / k_{0}$ \\
\hline \hline 1 & 2.186 & 2.187 & $\sim 10^{-8}$ \\
\hline 30 & 3.057 & 3.057 & $\sim 10^{-4}$ \\
\hline 60 & 3.124 & 3.124 & $\sim 10^{-6}$ \\
\hline 90 & 3.142 & 3.143 & $\sim 10^{-7}$ \\
\hline 120 & 3.150 & 3.151 & $\sim 10^{-6}$ \\
\hline
\end{tabular}

despite the lack of a rigorous physical background, the DCIT provided a sufficiently accurate mathematical expansion for the spatial Green's functions. This same rationale will be here used to extend the DCIT analysis of previous sections to study the propagation characteristics of covered 2-D guiding planar structures (such as that shown in Fig. 1, but covered with an upper electric wall). Next, some questions concerning the analysis of such structures, together with some results for bound and leaky line modes will be shown.

\section{A. Analysis}

It is well known that the spectral Green's functions of covered planar structures can be expressed in terms of even functions of the vertical wavenumber associated with each layer [16], which causes these functions to not have branch points in the $k_{\rho}$-plane, unlike what happens for open structures. Thus, the expansion of $\widetilde{G}$ in terms of complex exponentials can be carried out using the vertical wavenumber of any layer as the variable of the expansion. Specifically, the choice in this study will be to express the spectral Green's function in terms of the vertical wavenumber associated with the layer of maximum permittivity $k_{y, \max }$. The reason for choosing this variable is to enforce that all the propagative modes of the background waveguide lie on the real axis of the $k_{y, \max }$ complex plane (if another vertical wavenumber was used, these propagative modes will be located on the real, as well as on the imaginary axes). In consequence, all the expressions in Section II-B and C can now be used after substituting $k_{y 0}$ by $k_{y, \max }$. It should be noticed that, because of the evenness of the spectral Green's function, the natural strategy to now extract the poles is that in (7).

\section{B. Results}

Table IV shows a comparison between the SDA and DCIT results obtained for the propagation constant of the fundamental mode of a two-layer stripline. An excellent agreement has been found between both methods in the whole frequency range up to $120 \mathrm{GHz}$. The DCIT results have been obtained by using a sampling path of the $C_{1}$ type, but with the starting point of the upper segment almost reaching the origin of the complex plane since $k_{y, \max }\left(k_{x}=0\right) \simeq 0$. The number of exponentials used in each sampling segment has been adjusted to assure that the spectral Green's functions are approximated with at least four significant figures. 


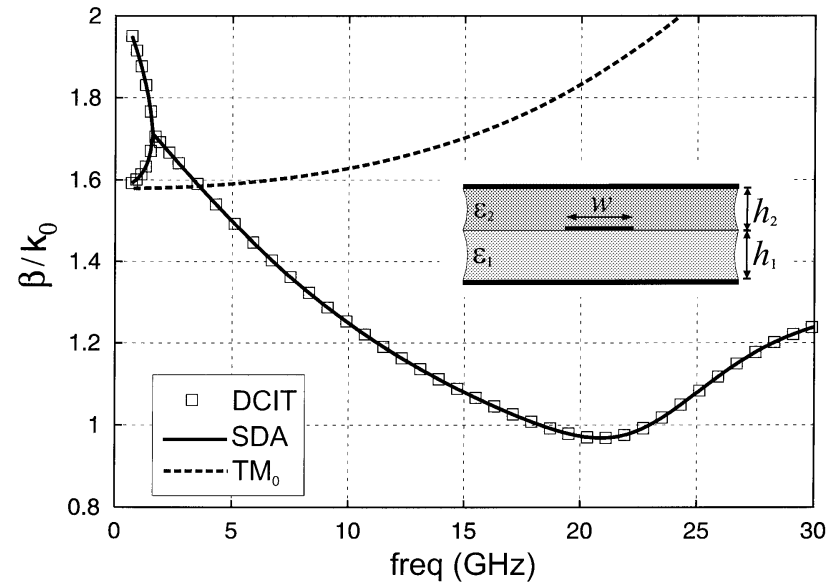

(a)

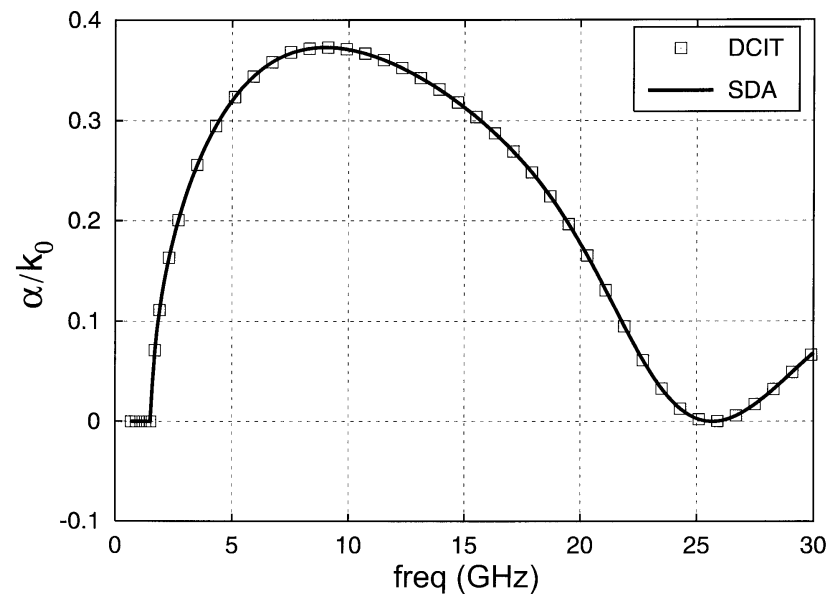

(b)

Fig. 8. Comparison between the results obtained for the normalized: (a) phase and (b) attenuation constants of a leaky mode for the covered structure previously analyzed in Table IV.

The DCIT has also been found to be efficient in studying the leaky regime in covered structures. Thus, Fig. 8(a) and (b) shows the results of the normalized phase and attenuation constants of a leaky mode present in the covered structure previously analyzed in Table IV. The lateral radiation of this leaky mode takes the form of only the dominant $\mathrm{TM}_{0}$ mode of the parallel-plate waveguide and its propagation constant has been computed by using a sampling path of the $C_{3}$ type.

\section{CONCLUSIONS}

This paper has presented a number of rules for the efficient application of the DCIT to approximate the kernel of the MPIE resulting from the analysis of 2-D planar transmission lines in layered media. It has been found that the path proposed thus far in the literature to sample the spectral Green's functions is not the most appropriate. Thus, new suitable sampling paths have been proposed for both the bound and leaky regimes. For bound modes, the use of these sampling paths allows to avoid the extraction of poles of the spectral Green's functions, whereas all the SW poles must be extracted when dealing with surface-leaky modes of the transmission line. For space-leaky modes, it has been found that some poles associated with LWs of the background waveguide must also be extracted. A new pole-extraction strategy was then presented in order to make feasible the extraction of the poles associated with these improper modes. The DCIT has also been applied to study covered 2-D planar transmission lines. A good numerical performance of this technique has also been found in this case.

\section{APPENDIX}

The spatial counterpart of the function employed to extract the poles (5) is given by

$$
G^{P}\left(\left|x-x^{\prime}\right| ; y, y^{\prime}\right)=\frac{1}{2 \pi} \sum_{p=1}^{N_{p}} R_{p}\left(y, y^{\prime}\right) \int_{-\infty}^{\infty} \frac{e^{-j k_{x}\left(x-x^{\prime}\right)}}{k_{y 0}-k_{y 0, p}} d k_{x}
$$

which renders the following integral to be computed:

$$
\begin{aligned}
I\left(k_{x 0}, k_{x p},\left|x-x^{\prime}\right|\right) & =\int_{-\infty}^{\infty} \frac{e^{-j k_{x}\left(x-x^{\prime}\right)}}{k_{y 0}-k_{y 0, p}} d k_{x} \\
& =\int_{-\infty}^{\infty} \frac{e^{-j k_{x}\left(x-x^{\prime}\right)}}{\sqrt{k_{x 0}^{2}-k_{x}^{2}}-k_{y 0, p}} d k_{x} .
\end{aligned}
$$

The integrand in (19) has branch points at $k_{x}= \pm k_{x 0}$ and poles at $k_{x}= \pm \sqrt{k_{x 0}^{2}-k_{y 0, p}^{2}}= \pm k_{x p}$ corresponding to either proper or improper modes. First, it will assumed that the pole under consideration corresponds to a proper SW mode. If the line mode under consideration is a bound mode, the integral in (19) must be performed along the real axis, whereas if the mode is a surface/space-leaky mode, the integration path must be such as that shown in Figs. 5(a) and 7(a). If $x-x^{\prime}>0$, the integrand in (19) decays exponentially to zero as $\Im\left(k_{x}\right) \rightarrow-\infty$ and, thus, the integration path can be continuously deformed downwards through the $k_{x}$-plane to give the equivalent paths shown in Fig. 9(a)-(c). It can be easily verified that, for the case $x-x^{\prime}<0$, analogous expressions as those presented in the following are achieved by similarly deforming the integration path upwards through the $k_{x}$-plane. For this reason, $x-x^{\prime}$ will be substituted next by $\left|x-x^{\prime}\right|$. Note that the equivalent integration paths in Fig. 9(a)-(c) consist on a CP that encircles a pole plus a steepest descent path (SDP). The integral in (19) can, therefore, be rewritten as

$$
\begin{aligned}
I\left(k_{x 0}, k_{x p},\left|x-x^{\prime}\right|\right)=I_{\mathrm{CP}} & \left(k_{x 0}, k_{x p},\left|x-x^{\prime}\right|\right) \\
& +I_{\mathrm{SDP}}\left(k_{x 0}, k_{x p},\left|x-x^{\prime}\right|\right) .
\end{aligned}
$$

The integral $I_{\mathrm{CP}}$ can be evaluated by a simple residue calculus yielding

$$
I_{\mathrm{CP}}\left(k_{x 0}, k_{x p},\left|x-x^{\prime}\right|\right)=2 \pi j \frac{k_{y 0, p}}{k_{x p}} e^{-j k_{x p}\left|x-x^{\prime}\right|}
$$

where $k_{x p}$ is the location of the enclosed pole in the $k_{x}$-plane; namely, $\Im\left(k_{x p}\right)<0$ for bound modes and $\Im\left(k_{x p}\right)>0$ for 


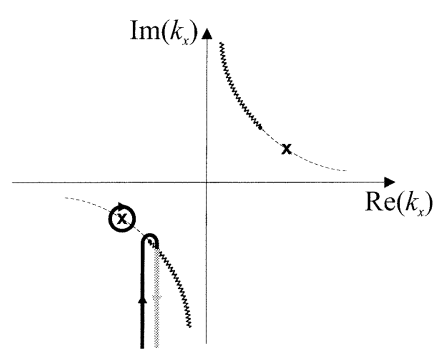

(a)

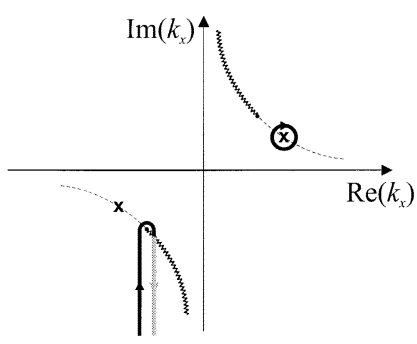

(b)

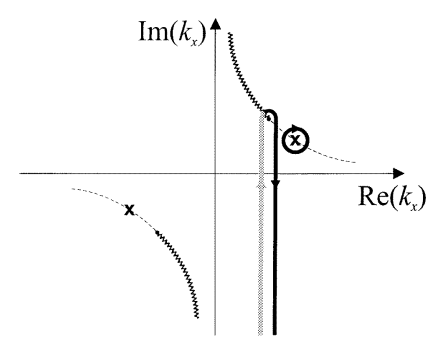

(c)

Fig. 9. Equivalent integration paths for: (a) bound, (b) surface-leaky, and (c) space-leaky modes. Black/grey colors denote that the integration path lies on the proper/improper sheet.

leaky modes, similar to [12]. Introducing the change of variable $k_{x}=k_{x 0}-j s, I_{\mathrm{SDP}}$ can be conveniently expressed as

$$
\begin{aligned}
& I_{\mathrm{SDP}}\left(k_{x 0}, k_{x p},\left|x-x^{\prime}\right|\right) \\
&= 2 j e^{-j k_{x 0}\left|x-x^{\prime}\right|} \operatorname{sign}\left\{\Re\left(k_{x 0}\right)\right\} \\
& \quad \times \int_{0}^{\infty} \frac{\sqrt{s^{2}+2 j k_{x 0} s}}{s^{2}+2 j k_{x 0} s-k_{y 0, p}^{2}} e^{-s\left|x-x^{\prime}\right|} d s
\end{aligned}
$$

where $\Im\left(\sqrt{s^{2}+2 j k_{x 0} s}\right)<0 ; \Im\left(k_{x 0}\right)<0$ for bound and surface-leaky modes and $\Im\left(k_{x 0}\right)>0$ for space-leaky modes. For $s \gg\left|k_{x 0}\right|$, it is clear that $\operatorname{sign}\left\{\Re\left(k_{x 0}\right)\right\} \sqrt{s^{2}+2 j k_{x 0} s} \approx$ $-\left(s+j k_{x 0}\right)$. This asymptotic behavior can now be extracted from $I_{\mathrm{SDP}}$ to give

$$
\begin{aligned}
& I_{\mathrm{SDP}}\left(k_{x 0}, k_{x p},\left|x-x^{\prime}\right|\right) \\
& =2 j e^{-j k_{x 0}\left|x-x^{\prime}\right|}\left[I_{1}\left(k_{x 0}, k_{x p},\left|x-x^{\prime}\right|\right)\right. \\
& \left.+I_{2}\left(k_{x 0}, k_{x p},\left|x-x^{\prime}\right|\right)\right]
\end{aligned}
$$

where

$$
\begin{aligned}
& I_{1}\left(k_{x 0}, k_{x p},\left|x-x^{\prime}\right|\right) \\
& =\int_{0}^{\infty} \frac{s+j k_{x 0}+\operatorname{sign}\left\{\Re\left(k_{x 0}\right)\right\} \sqrt{s^{2}+2 j k_{x 0} s}}{s^{2}+2 j k_{x 0} s-k_{y 0, p}^{2}} \\
& \quad \times e^{-s\left|x-x^{\prime}\right|} d s \\
& I_{2}\left(k_{x 0}, k_{x p},\left|x-x^{\prime}\right|\right) \\
& =\int_{0}^{\infty} \frac{-\left(s+j k_{x 0}\right)}{s^{2}+2 j k_{x 0} s-k_{y 0, p}^{2}} e^{-s\left|x-x^{\prime}\right|} d s .
\end{aligned}
$$

The fraction in the integrand of (25) can be split into a sum of two fractions that give rise to two integrals having closed-form expressions [20] as follows:

$$
\begin{aligned}
- & 2 I_{2}\left(k_{x 0}, k_{x p},\left|x-x^{\prime}\right|\right) \\
& =\int_{0}^{\infty} \frac{e^{-s\left|x-x^{\prime}\right|}}{s+s_{1}} d s+\int_{0}^{\infty} \frac{e^{-s\left|x-x^{\prime}\right|}}{s+s_{2}} d s \\
& =e^{s_{1}\left|x-x^{\prime}\right|} E_{1}\left(s_{1}\left|x-x^{\prime}\right|\right)+e^{s_{2}\left|x-x^{\prime}\right|} E_{1}\left(s_{2}\left|x-x^{\prime}\right|\right)
\end{aligned}
$$

where $s_{1}=j\left(k_{x 0}-k_{x p}\right), s_{2}=j\left(k_{x 0}+k_{x p}\right)$, and $E_{1}(\cdot)$ is the exponential integral function. The remaining integral $I_{1}$ has to be computed numerically, although it is rapidly convergent since the asymptotic behavior has been extracted out. Thus, (24) can be quickly computed with low computational effort by using, for instance, a Gauss-Kronrod adaptive quadrature scheme.

In the case of poles associated with improper real modes in (19), the poles of $\widetilde{G}^{P}$ in the $k_{x}$-plane lie on the hyperbola defined by $\Im\left(k_{y 0}\right)=0$ (just as the SW poles), although located on the improper sheet and, thus, they are never captured by the integration path. Consequently, for improper real poles, only the SDP contribution is present. Concerning the extraction of LW modes, the CP contribution has to be included as long as the corresponding pole is enclosed as the SDA integration path is continuously deformed to an SDP. It is clear that this term will never be present when searching for bound or surface-leaky modes, but, in the case of space-leaky modes, a former study of the SDA integration path together with the location of the poles to infer whether it is (or not) present becomes necessary.

The final quasi-analytical expression for the inverse transform of $\widetilde{G}^{P}$ can then be written as

$$
\begin{aligned}
& G_{p}(\left.\left|x-x^{\prime}\right| ; y, y^{\prime}\right) \\
&= \sum_{p=1}^{N_{p}} \frac{j R_{p}\left(y, y^{\prime}\right)}{\pi} \\
& \quad \times\left\{\left[\delta_{p} \pi \frac{k_{y 0, p}}{k_{x p}}+\frac{1}{2} E_{1}\left(s_{1}\left|x-x^{\prime}\right|\right)\right] e^{-j k_{x p}\left|x-x^{\prime}\right|}\right. \\
& \quad+\frac{1}{2} E_{1}\left(s_{2}\left|x-x^{\prime}\right|\right) e^{j k_{x p}\left|x-x^{\prime}\right|} \\
&\left.\quad-I_{1}\left(k_{x 0}, k_{x p},\left|x-x^{\prime}\right|\right) e^{-j k_{x 0}\left|x-x^{\prime}\right|}\right\}
\end{aligned}
$$

where $\delta_{p}=1(=0)$ if the pole is (not) enclosed according to the above discussion.

\section{REFERENCES}

[1] D. G. Fang, J. J. Yang, and G. Y. Delisle, "Discrete image theory for horizontal electric dipoles in a multilayered medium," Proc. Inst. Elect. Eng., pt. H, vol. 135, pp. 297-303, Oct. 1988.

[2] Y. L. Chow, J. J. Yang, D. G. Fang, and G. E. Howard, "A closed-form spatial Green's function for the thick microstrip substrate," IEEE Trans. Microwave Theory Tech., vol. 39, pp. 588-592, Mar. 1991.

[3] M. I. Aksun and R. Mittra, "Derivation of closed-form Green's functions for a general microstrip geometry," IEEE Trans. Microwave Theory Tech., vol. 40, pp. 2055-2061, Nov. 1992. 
[4] R. A. Kipp and C. H. Chan, "Complex image method for sources in bounded regions of multilayer structures," IEEE Trans. Microwave Theory Tech., vol. 42, pp. 860-865, May 1994.

[5] — - "A complex image method for the vertical component of the magnetic potential of a horizontal dipole in layered media," in Proc. IEEE AP-S Conf., vol. 2, Seattle, WA, June 1994, pp. 1366-1369.

[6] M. I. Aksun, "A robust approach for the derivation of closed-form Green's functions," IEEE Trans. Microwave Theory Tech., vol. 44, pp. 651-658, May 1996.

[7] J. Bernal, F. Medina, and M. Horno, "Quick quasi-TEM analysis of multiconductor transmission lines with rectangular cross section," IEEE Trans. Microwave Theory Tech., vol. 45, pp. 1619-1626, Sept. 1997.

[8] E. A. Soliman, P. Pieters, E. Beyne, and G. A. E. Vandenbosh, "Numerically efficient method for multislot transmission lines in layered media-Application to multislot lines in MCM-D technology," IEEE Trans. Microwave Theory Tech., vol. 47, pp. 1782-1787, Sept. 1999.

[9] J. Bernal, F. Medina, R. R. Boix, and M. Horno, "Fast full-wave analysis of multistrip transmission lines based on MPIE and complex image theory," IEEE Trans. Microwave Theory Tech., vol. 48, pp. 445-452, Mar. 2000.

[10] J. Bernal, F. Medina, and R. R. Boix, "Full-wave analysis of nonplanar transmition lines on layered medium by means of MPIE and complex image theory," IEEE Trans. Microwave Theory Tech., vol. 49, pp. 177-185, Jan. 2001.

[11] E. A. Soliman, G. A. E. Vandenbosh, E. Beyne, and R. P. Mertens, "Full-wave analysis of multiconductor multislot planar guiding structures in layered media," IEEE Trans. Microwave Theory Tech., vol. 51, pp. 874-886, Mar. 2003.

[12] J. Bernal, F. Mesa, and F. Medina, "2-D analysis of leakage in printedcircuit lines using discrete complex-images technique," IEEE Trans. Microwave Theory Tech., vol. 50, pp. 1895-1900, Aug. 2002.

[13] F. Mesa, C. Di Nallo, and D. R. Jackson, "The theory of surface-wave and space-wave leaky-mode excitation on microstrip lines," IEEE Trans. Microwave Theory Tech., vol. 47, pp. 207-215, Feb. 1999.

[14] N. V. Shuley, R. R. Boix, F. Medina, and M. Horno, "On the fast approximation of Green's functions in MPIE formulations for planar layered media," IEEE Trans. Microwave Theory Tech., vol. 50, pp. 2185-2191, Sept. 2002.

[15] K. A. Michalski and D. Zheng, "Electromagnetic scattering and radiation by surfaces of arbitrary shape in layered media-Part I: Theory," IEEE Trans. Antennas Propagat., vol. 38, pp. 335-344, Mar. 1990.

[16] L. B. Felsen and N. Marcuvitz, Radiation and Scattering of Waves, 2nd ed. Piscataway, NJ: IEEE Press, 1995.

[17] T. K. Sarkar and O. Pereira, "Using the matrix pencil method to estimate the parameters of a sum of complex exponentials," IEEE Antennas Propagat. Mag., vol. 37, pp. 48-55, Feb. 1995.

[18] J. L. Tsalamengas, "Rapidly converging direct singular integral-equation techniques in the analysis of open microstrip lines on layered substrates," IEEE Trans. Microwave Theory Tech., vol. 49, pp. 555-559, Mar. 2001.

[19] J. J. Yang, Y. L. Chow, G. E. Howard, and D. G. Fang, "Complex images of an electric dipole in homogeneous and layered dielectrics between two ground planes," IEEE Trans. Microwave Theory Tech., vol. 40, pp. 595-600, Mar. 1992.

[20] M. Abramowitz and I. A. Stegun, Handbook of Mathematical Functions. New York: Dover, 1972.

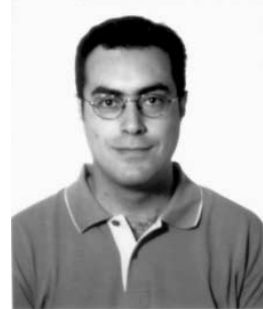

Raúl Rodríguez-Berral was born in Casariche, Seville, Spain, on August 1978. He received the Licenciado degree in physics from the Universidad de Sevilla, Seville, Spain, in 2001, and is currently working toward the Ph.D. degree at the Universidad de Sevilla.

In January 2002, he joined the Departamento de Física Aplicada 1, Universidad de Sevilla.

Mr. Rodríguez-Berral was the recipient of a Spanish Ministry of Science and Technology Scholarship.

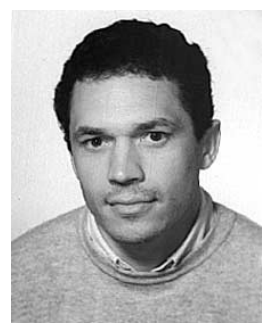

Francisco Mesa (M'94) was born in Cádiz, Spain, in April 1965. He received the Licenciado and Doctor degrees from the Universidad de Sevilla, Seville, Spain, in 1989 and 1991, respectively, both in physics.

$\mathrm{He}$ is currently an Associate Professor with the Departamento de Física Aplicada 1, Universidad de Sevilla. His research interest focuses on electromagnetic propagation/radiation in planar lines with general anisotropic materials.

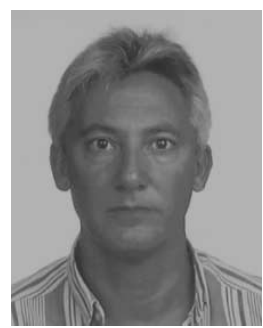

Francisco Medina (M'90-SM'01) was born in Puerto Real, Cádiz, Spain, in November 1960. He received the Licenciado and Doctor degrees from the Universidad de Sevilla, Seville, Spain, in 1983 and 1987, respectively, both in physics.

From 1986 to 1987, he spent the academic year with the Laboratoire de Microondes de l'ENSEEIHT, Toulouse, France. From 1985 to 1989, he was a Profesor Ayudante (Assistant Professor) with the Departamento de Electrónica y Electromagnetismo, Universidad de Sevilla, and since 1990, he has been a Profesor Titular (Associate Professor) of electromagnetism. He is also currently Head of the Grupo de Microondas, Universidad de Sevilla. His research interest includes analytical and numerical methods for guidance, resonant and radiating structures, passive planar circuits, and the influence on these circuits of anisotropic materials. He is reviewer of a number of international scientific and technical journals and local conferences.

Dr. Medina was a member of the Technical Program Committee (TPC) of the 23rd European Microwave Conference, Madrid, Spain, 1993, the TPC of ISRAMT, Malaga, Spain, 1999, and the TPC of the Microwaves Symposiun, Tetouan, Morocco, 2000. He is on the Editorial Board of the IEEE TRAnsactions on MicrowaVe TheORY and TeChNiques. He has been a reviewer for other IEEE and Institution of Electrical Engineers (IEE), U.K., publications. He was the recipient of a Ministerio de Educación y Ciencia/Ministere de la Recherche et la Technologie Scholarship. 